\title{
Impacts of climate change on air pollution levels in the northern hemisphere with special focus on Europe and the Arctic
}

\author{
G. B. Hedegaard, J. Brandt, J. H. Christensen, L. M. Frohn, C. Geels, K. M. Hansen, T. Hvid, and C. A. Skjøth \\ Aarhus University, National Environmental Research Institute, Department of Atmospheric Environement, Roskilde, Denmark \\ (jbr@dmu.dk,+45-(0)4630-1214)
}

The response of a selected number of chemical species is inspected with respect to climate change. The coupled Atmosphere-Ocean General Circulation Model ECHAM4-OPYC3 is providing meteorological fields for the Chemical long-range Transport Model DEHM. Several selected decades (e.g. 1990s and 2090s) are inspected. The 1990s are used as a reference and validation period. In this decade an evaluation of the output from the DEHM model with ECHAM4-OPYC3 meteorology input data is carried out. The model results are tested against similar model simulations with MM5 meteorology and against observations from the EMEP monitoring sites in Europe.

The dominating impacts from climate change on a large number of the chemical species are related to the predicted temperature increase. Throughout the 21st century the ECHAM4-OPYC3 projects a global mean temperature increase of $3 \mathrm{~K}$ with local maxima up to $11 \mathrm{~K}$ in the Arctic winter based on the IPCC A2 emission scenario. As a consequence of this temperature increase, the temperature dependent biogenic emission of isoprene is predicted to increase significantly over land by the DEHM model. This leads to an increase in the $\mathrm{O} 3$ production and together with an increase in water vapour to an increase in the number of free $\mathrm{OH}$ radicals. Furthermore this increase in the number of $\mathrm{OH}$ radicals contributes to a significant change in the typical life time of many species, since $\mathrm{OH}$ are participating in a large number of chemical reactions. It is e.g. found that more SO4 will be present in the future over the already polluted areas and this increase can be explained by an enhanced conversion of SO2 to $\mathrm{SO} 4$. 